\title{
On the trace of powers of square matrices
}

\author{
Kailash M. Patil (10 \\ Department of Mathematics, Dharmsinh Desai University, Nadiad, Gujarat, INDIA 387001
}

\begin{abstract}
Using Cayley-Hamilton equation for matrices, we obtain a simple formula for trace of powers of a square matrix. The formula becomes simpler in particular cases. As a consequence, we also demonstrate the formula for trace of negative powers of a matrix.
\end{abstract}

Mathematics Subject Classification (2020). 15A24, 15A45

Keywords. trace of a matrix, powers of a matrix, Cayley-Hamilton theorem, spectral radius

\section{Introduction}

With the advancement of highly complex computer network topologies and eternally growing number of nodes in the existing networks, certain applications require to find the number of cliques in the graph of a given network. Using the adjacency matrix $A$ of the graph, one clique of vertices $v_{1}, v_{2}, v_{3}$ contributes the 2 to each of the $a_{11}, a_{22}, a_{33}$. Thus the count of cliques will be $\frac{\operatorname{Tr}\left(A^{3}\right)}{6}[2]$. In [6], an identity involving the Eulerian congruence on trace of powers of integer matrices modulo $p^{r}$ is obtained, where $p$ is prime, and $r \in \mathbb{N}$. [4] makes a short survey of related results. For a square matrix $A=\left[a_{i j}\right]$, the trace of $A$ denoted by $\operatorname{Tr}(A)$, is the sum of main diagonal entries of $A$, that is $\operatorname{Tr}(A)=\sum_{i} a_{i i}$. [5] obtains the formula of computation of the eigenvalue with maximum modulus of a matrix using the trace of its higher powers. Our formula thus contributes to finding the spectral radius of a matrix. [1] also developes the similar formula for $n^{\text {th }}$ power of a $2 \times 2$ matrix. Our formula is a general one and does not require computation of entries of $n^{\text {th }}$ power.

The current paper is in the sequel of [3], wherein we have obtained the formula for the sum of the powers of matrices and its consequences. In Section 2, we set the required notations and recall the terminology. We also state the main result Theorem 2.1. The simplification of the long computations in the proofs are achieved by introducing the functions $l_{m}\left(n, k_{0}, k_{1}, \ldots, k_{m-2}\right)$ used for finding trace of $n^{\text {th }}$ power of an $m \times m$ matrix $A$. The introduction of $l_{m}(\cdot)$ is motivated by the list of expression of $\operatorname{Tr}\left(A^{n}\right)$ for a $3 \times 3$ matrix $A$ for first few powers of $A$. The jargon of notations, as one will be convinced, is used only for the proof to be simplified. However, the actual application of our formulae to real computation does not require much of knowledge except the definition of the Trace and a couple of related definitions. The proof of the main theorem is discussed in Section 3. In fact, a technical formula (3.1) for $l_{m}(\cdot)$ is obtained in a series of Lemmas using Mathematical Induction. Very important and useful particular cases are discussed

Email address: kailashpatil2111@gmail.com Received: 29.04.2019; Accepted: 12.04.2020 
in Section 4. Finally the formula for the trace of negative powers of nonsingular matrices is demonstrated in Section 5. To maintain the brevity, we restrict ourselves to $2 \times 2$ matrices for negative powers. However, we should impress upon the reader that this restrictions can easily be done away with.

\section{Main result}

In what follows, $A=\left[a_{i j}\right]$ denotes an $m \times m$ matrix. For any integer $1 \leq k \leq m$ and the integers $1 \leq i_{1} \leq i_{2} \leq i_{3} \leq \cdots \leq i_{k} \leq m$, the determinant of the $k \times k$ submatrix obtained by removing all rows except $i_{1}, i_{2}, i_{3}, \ldots, i_{k}$ rows and $i_{1}, i_{2}, i_{3}, \ldots, i_{k}$ columns is called a principal minor of $A$ of order $k$, thereby obtaining $\left(\begin{array}{c}m \\ k\end{array}\right)$ minors. We denote their sum as $S_{k}(A)$ or for $S_{k}$ for brevity whenever there is no confusion. Thus, $S_{1}$ will become the trace of the given matrix and $S_{n}$ will be the determinant of $A$.

The characteristic equation of $A$ is given by

$$
\operatorname{det}(A-\lambda I)=0,
$$

where $I$ is $m \times m$ identity matrix. The roots of the characteristic equation are called the characteristic roots of $A$. We shall denote them by $\lambda_{1}, \lambda_{2}, \ldots, \lambda_{m}$.

The motivation for defining ingredients required for the formula of trace of powers of $A$ lies in the analysis of a $3 \times 3$ matrix, and hence, for time being, $A$ will denote a $3 \times 3$ matrix.

The characteristic equation of $A$ is

$$
\lambda^{3}-S_{1} \lambda^{2}+S_{2} \lambda-S_{3}=0
$$

where $S_{1}=\operatorname{Tr}(A)=\lambda_{1}+\lambda_{2}+\lambda_{3}=\sum_{i=1}^{3} a_{i i}, S_{2}=\sum_{i \neq j} \lambda_{i} \lambda_{j}$ and $S_{3}=\lambda_{1} \lambda_{2} \lambda_{3}=\operatorname{det}(A)$.

By the Cayley-Hamilton theorem, we have $A^{3}-S_{1} A^{2}+S_{2} A-S_{3} I=0$. This, in turn, implies the following for $n \in \mathbb{N}$.

$$
A^{n+3}-S_{1} A^{n+2}+S_{2} A^{n+1}-S_{3} A^{n}=0 .
$$

Applying the trace, a linear operator, on (2.1) gives a recursive relation,

$$
\operatorname{Tr}\left(A^{n+3}\right)=S_{1} \operatorname{Tr}\left(A^{n+2}\right)-S_{2} \operatorname{Tr}\left(A^{n+1}\right)+S_{3} \operatorname{Tr}\left(A^{n}\right),
$$

which is central to this note. Observe that

$$
\begin{aligned}
\operatorname{Tr}\left(A^{2}\right) & =\lambda_{1}^{2}+\lambda_{2}^{2}+\lambda_{3}^{2}=\left(\lambda_{1}+\lambda_{2}+\lambda_{3}\right)^{2}-2\left(\lambda_{1} \lambda_{2}+\lambda_{2} \lambda_{3}+\lambda_{1} \lambda_{3}\right) \\
& =S_{1}^{2}-2 S_{2} .
\end{aligned}
$$

Putting particular values of $n \in Z_{+} \cup\{0\}$ in (2.2) and simplifying, we have the following.

$$
\begin{aligned}
& \operatorname{Tr}\left(A^{3}\right)=S_{1}^{3}-3 S_{1} S_{2}+3 S_{3} . \\
& \operatorname{Tr}\left(A^{4}\right)=S_{1}^{4}-4 S_{1}^{2} S_{2}+2 S_{2}^{2}+4 S_{1} S_{3} . \\
& \operatorname{Tr}\left(A^{5}\right)=S_{1}^{5}-5 S_{1}^{3} S_{2}+5 S_{1} S_{2}^{2}+\left(5 S_{1}^{2}-5 S_{2}\right) S_{3} . \\
& \operatorname{Tr}\left(A^{6}\right)=S_{1}^{6}-6 S_{1}^{4} S_{2}+9 S_{1}^{2} S_{2}^{2}-2 S_{2}^{3}+\left(6 S_{1}^{3}-12 S_{1} S_{2}\right) S_{3}+3\left(S_{3}\right)^{2} . \\
& \operatorname{Tr}\left(A^{7}\right)=S_{1}^{7}-7 S_{1}^{5} S_{2}+14 S_{1}^{3} S_{2}^{2}-7 S_{1} S_{2}^{3}+\left(7 S_{1}^{4}-21 S_{1}^{2} S_{2}+7 S_{2}^{2}\right) S_{3}+\left(7 S_{1}\right) S_{3}^{2} .
\end{aligned}
$$

It is quite apparent that the complexity of the formula increases as the power increases. Well within the ninth power, the formula really becomes highly involved.

$$
\begin{aligned}
\operatorname{Tr}\left(A^{9}\right)= & S_{1}^{9}-9 S_{1}^{7} S_{2}+27 S_{1}^{5} S_{2}^{2}-30 S_{1}^{3} S_{2}^{3}+9 S_{1} S_{2}^{4}+\left(9 S_{1}^{6}-45 S_{1}^{4} S_{2}+54 S_{1}^{2} S_{2}^{2}-9 S_{2}^{3}\right) S_{3} \\
& +\left(18 S_{1}^{3}-27 S_{1} S_{2}\right) S_{3}^{2}+3 S_{3}^{3}
\end{aligned}
$$




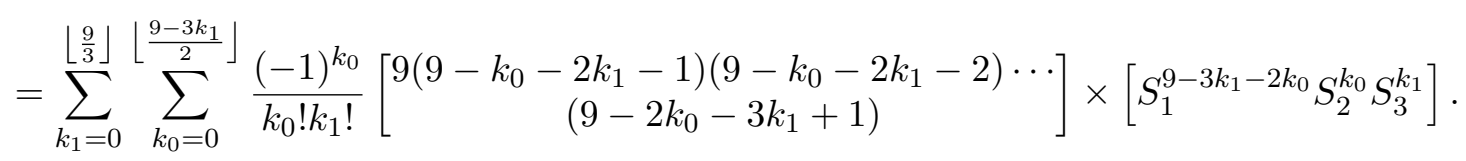

Before we conclude the general formula for $\operatorname{Tr}\left(A^{n}\right)$, we define

$$
l_{3}\left(n, k_{0}, k_{1}\right)= \begin{cases}\frac{1}{k_{0} ! k_{1} !} n\left(n-k_{0}-2 k_{1}-1\right)\left(n-k_{0}-2 k_{1}-2\right) & \\ \times\left(n-k_{0}-2 k_{1}-3\right) \cdots\left(n-2 k_{0}-3 k_{1}+1\right), & \text { if } k_{0}+k_{1} \geq 2 ; \\ n, & \text { if } k_{0}+k_{1}=1 \\ 1, & \text { if } k_{0}+k_{1}=0 .\end{cases}
$$

The above definition is applied only when each $k_{i} \geq 0$. In the course of different order of matrices we get different $l_{m}\left(n, k_{0}, k_{1}, \cdots, k_{m-2}\right)$. Throughout this note, we adopt the convention that if at least one $k_{i}<0$, then we define $l_{m}\left(n, k_{0}, k_{1}, \cdots, k_{m-2}\right)=0$. As a consequence, In general, for $m \times m$ matrix

$$
l_{m}\left(n, k_{0}, k_{1}, \ldots, k_{m-2}\right)=\frac{n}{k_{0} ! k_{1} ! \cdots ! k_{m-2} !}\left[\begin{array}{c}
\left(n-k_{0}-2 k_{1}-\cdots-(m-1) k_{m-2}-1\right) \\
\times\left(n-k_{0}-2 k_{1}-\cdots-(m-1) k_{m-2}-2\right) \\
\times \cdots \\
\times\left(n-2 k_{0}-3 k_{1}-\cdots-m k_{m-2}+1\right)
\end{array}\right] .
$$

To shorten the displayed identities, when $n, k_{0}, k_{1}, \ldots, k_{m-2}$ are already mentioned in the summation, we write $l_{m}$ for $l_{m}\left(n, k_{0}, k_{1}, \ldots, k_{m-2}\right)$. Our main result in terms of a function $l_{m}$ is Theorem 2.1.

Theorem 2.1. For a $m \times m$ matrix $A=\left[a_{i j}\right]$, we have

$$
\begin{aligned}
\operatorname{Tr}\left(A^{n}\right)= & \sum_{k_{j} \geq 0} \sum_{k_{0}=0}^{\left\lfloor\frac{n-3 k_{1}-4 k_{2}-\cdots-m k_{m-2}}{2}\right\rfloor}(-1)^{k_{0}+k_{2}+k_{4}+\cdots k_{\left\lfloor\frac{m-2}{2}\right\rfloor}} l_{m} \\
& \times\left[S_{1}^{n-2 k_{0}-3 k_{1}-4 k_{2}-\cdots-m k_{m-2}} S_{2}^{k_{0}} S_{3}^{k_{1}} S_{4}^{k_{2}} \cdots S_{m-1}^{k_{m-3}} S_{m}^{k_{m-2}}\right] .
\end{aligned}
$$

For a nonsingular $m \times m$ matrix $A$, one observes that

$$
\begin{aligned}
S_{1}\left(A^{-1}\right) & =\operatorname{Tr}\left(A^{-1}\right)=\frac{1}{\lambda_{1}}+\frac{1}{\lambda_{2}}+\cdots+\frac{1}{\lambda_{m}}=\frac{S_{m-1}(A)}{S_{m}(A)} . \\
S_{2}\left(A^{-1}\right) & =\sum_{\substack{i, j=1 \\
i<j}}^{m} \frac{1}{\lambda_{i} \lambda_{j}}=\frac{S_{m-2}(A)}{S_{m}(A)} . \\
\cdots & =\cdots \\
S_{m-1}\left(A^{-1}\right) & =\sum_{i=1}^{m} \frac{1}{\lambda_{1} \lambda_{2} \cdots \lambda_{i-1} \lambda_{i+1} \cdots \lambda_{m}}=\frac{S_{1}(A)}{S_{m}(A)} . \\
S_{m}\left(A^{-1}\right) & =\frac{1}{\lambda_{1} \lambda_{2} \cdots \lambda_{m}}=\frac{1}{S_{m}(A)} .
\end{aligned}
$$

Using all this, and replacing $A$ by $A^{-1}$ in the Theorem 2.1 , the following is at once.

Theorem 2.2. For a $m \times m$ nonsingular matrix $A=\left[a_{i j}\right]$, we have

$$
\begin{aligned}
\operatorname{Tr}\left(A^{-n}\right)= & \frac{1}{[\operatorname{det}(A)]^{n}} \sum_{k_{j} \geq 0}^{\left\lfloor\frac{n-3 k_{1}-4 k_{2}-\cdots-m k_{m-2}}{2}\right\rfloor} \sum_{k_{0}=0}^{2}(-1)^{k_{0}+k_{2}+k_{4}+\cdots k_{\left\lfloor\frac{m-2}{2}\right\rfloor}} l_{m} \\
& \times\left[S_{1}^{k_{m-3}} S_{2}^{k_{m-4}} \cdots S_{m-1}^{n-2 k_{0}-3 k_{1}-\cdots-m k_{m-2}} S_{m}^{k_{0}+2 k_{1}+3 k_{2}+\cdots+(m-1) k_{m-2}}\right] .
\end{aligned}
$$




\section{Proof of the main theorem}

In order to prove the main theorem, we first prove the following.

$$
\begin{aligned}
l_{m}\left(n, k_{0}, k_{1}, \ldots, k_{m-2}\right)= & l_{m}\left(n-1, k_{0}, k_{1}, \ldots, k_{m-2}\right) \\
& +\sum_{i=2}^{m} l_{m}\left(n-i, k_{0}, k_{1}, \ldots, k_{i-2}-1, \ldots, k_{m-2}\right) .
\end{aligned}
$$

We establish (3.1) by applying mathematical induction on the order of the matrix $A=\left[a_{i j}\right]$. The proof is divided into a couple of Lemmas.

Lemma 3.1. $l_{2}\left(n, k_{0}\right)=l_{2}\left(n-1, k_{0}\right)+l_{2}\left(n-2, k_{0}-1\right)$.

Proof. Since the cases $k_{0}=0$ and $k_{0}=1$ are trivial, we can assume that $k_{0} \geq 2$. Now

$$
\begin{aligned}
l_{2}\left(n-1, k_{0}\right)+l_{2}\left(n-2, k_{0}-1\right)= & \frac{(n-1)\left(n-k_{0}-2\right)\left(n-k_{0}-3\right) \cdots\left(n-2 k_{0}\right)}{k_{0} !} \\
& +\frac{(n-2)\left(n-k_{0}-2\right)\left(n-k_{0}-3\right) \cdots\left(n-2 k_{0}+1\right)}{\left(k_{0}-1\right) !} \\
= & \frac{\left(n-k_{0}-2\right)\left(n-k_{0}-3\right) \cdots\left(n-2 k_{0}+1\right)}{\left(k_{0}-1\right) !} \\
& \times\left[\frac{(n-1)\left(n-2 k_{0}\right)}{k_{0}}+n-2\right] \\
= & \frac{\left(n-k_{0}-2\right)\left(n-k_{0}-3\right) \cdots\left(n-2 k_{0}+1\right)}{\left(k_{0}-1\right) !} \\
& \times\left[\frac{\left.n^{2}-2 n k_{0}-n+2 k_{0}+n k_{0}-2 k_{0}\right]}{k_{0}}\right] \\
= & \frac{\left(n-k_{0}-2\right)\left(n-k_{0}-3\right) \cdots\left(n-2 k_{0}+1\right)}{\left(k_{0}-1\right) !} \\
& \times\left[\frac{n\left(n-k_{0}-1\right)}{k_{0}}\right] \\
= & l_{2}\left(n, k_{0}\right) .
\end{aligned}
$$

Lemma 3.2. $l_{3}\left(n, k_{0}, k_{1}\right)=l_{3}\left(n-1, k_{0}, k_{1}\right)+l_{3}\left(n-2, k_{0}-1, k_{1}\right)+l_{3}\left(n-3, k_{0}, k_{1}-1\right)$.

Proof. If $k_{1}=0$, then $l_{3}\left(n, k_{0}, k_{1}\right)=l_{2}\left(n, k_{0}\right)$ and $l_{3}\left(n-3, k_{0}, k_{1}-1\right)=0$. Consequently, our case reduces to the Lemma 3.1. For $k_{0}=0$ and $k_{1} \geq 1$, we have,

$$
\begin{aligned}
\text { R.H.S. }= & l_{3}\left(n-1,0, k_{1}\right)+l_{3}\left(n-3,0, k_{1}-1\right) \\
= & \frac{(n-1)\left(n-2 k_{1}-2\right)\left(n-2 k_{1}-3\right) \cdots\left(n-3 k_{1}\right)}{k_{1} !} \\
& +\frac{(n-3)\left(n-2 k_{1}-2\right)\left(n-2 k_{1}-3\right) \cdots\left(n-3 k_{1}+1\right)}{\left(k_{1}-1\right) !} \\
= & \frac{\left(n-2 k_{1}-2\right)\left(n-2 k_{1}-3\right) \cdots\left(n-3 k_{1}+1\right)}{\left(k_{1}-1\right) !}\left[\frac{(n-1)\left(n-3 k_{1}\right)}{k_{1}}+n-3\right] \\
= & \frac{\left(n-2 k_{1}-2\right)\left(n-2 k_{1}-3\right) \cdots\left(n-3 k_{1}+1\right)}{\left(k_{1}-1\right)}\left[\frac{n\left(n-2 k_{1}-1\right)}{k_{1}}\right] \\
= & l_{3}\left(n, 0, k_{1}\right) \\
= & \text { L.H.S. }
\end{aligned}
$$


Since the case $k_{0}=0=k_{1}$ is trivial, we assume now $k_{0}, k \geq 1$.

$$
\begin{aligned}
\text { R.H.S. }= & l_{3}\left(n-1, k_{0}, k_{1}\right)+l_{3}\left(n-2, k_{0}-1, k_{1}\right)+l_{3}\left(n-3, k_{0}, k_{1}-1\right) \\
= & \frac{(n-1)\left(n-k_{0}-2 k_{1}-2\right)\left(n-k_{0}-2 k_{1}-3\right) \cdots\left(n-2 k_{0}-3 k_{1}\right)}{k_{0} ! k_{1} !} \\
& +\frac{(n-2)\left(n-k_{0}-2 k_{1}-2\right)\left(n-k_{0}-2 k_{1}-3\right) \cdots\left(n-2 k_{0}-3 k_{1}+1\right)}{\left(k_{0}-1\right) ! k_{1} !} \\
& +\frac{(n-3)\left(n-k_{0}-2 k_{1}-2\right)\left(n-k_{0}-2 k_{1}-3\right) \cdots\left(n-2 k_{0}-3 k_{1}+1\right)}{k_{0} !\left(k_{1}-1\right) !} \\
= & \frac{\left(n-k_{0}-2 k_{1}-2\right)\left(n-k_{0}-2 k_{1}-3\right) \cdots\left(n-2 k_{0}-3 k_{1}+1\right)}{\left(k_{0}-1\right) !\left(k_{1}-1\right) !} \\
& \times\left[\frac{(n-1)\left(n-2 k_{0}-3 k_{1}\right)}{k_{0} k_{1}}+\frac{n-2}{k_{1}}+\frac{n-3}{k_{0}}\right] \\
= & \frac{\left(n-k_{0}-2 k_{1}-2\right)\left(n-k_{0}-2 k_{1}-3\right) \cdots\left(n-2 k_{0}-3 k_{1}+1\right)}{\left(k_{0}-1\right) !\left(k_{1}-1\right) !} \\
& \times\left[\frac{n\left(n-k_{0}-2 k_{1}-1\right)}{k_{0} k_{1}}\right] \\
= & l_{3}\left(n, k_{0}, k_{1}\right) \\
= & L . H . S .
\end{aligned}
$$

Lemma 3.3. As an induction hypothesis, assume that

$$
\begin{aligned}
l_{t}\left(n, k_{0}, k_{1}, k_{2}, \ldots, k_{t-2}\right)= & l_{t-1}\left(n-1, k_{0}, k_{1}, k_{2}, \ldots, k_{t-2}\right) \\
& +\sum_{i=2}^{t} l_{t-1}\left(n-i, k_{0}, k_{1}, k_{2}, \ldots, k_{i-2}-1, \ldots, k_{t-2}\right)
\end{aligned}
$$

for $t \leq m-1$. Then

$$
\begin{aligned}
l_{m}\left(n, k_{0}, \ldots, k_{m-2}\right)= & l_{m}\left(n-1, k_{0}, \ldots, k_{m-2}\right) \\
& +\sum_{i=2}^{m} l_{m}\left(n-i, k_{0}, k_{1}, \ldots, k_{i-2}-1, \ldots, k_{m-2}\right) .
\end{aligned}
$$

Proof. If $k_{m-2}=0$, then $l_{m}\left(n, k_{0}, \ldots, k_{m-2}\right)=l_{m-1}\left(n, k_{0}, \ldots, k_{m-3}\right)$

and $l_{m}\left(n, k_{0}, k_{1}, \ldots, k_{m-2}-1\right)=0$. Therefore, (3.3) follows from the Induction Hypothesis (3.2). Let $k_{j}=0$ for some $0 \leq j \leq m-1$. Then

$$
\begin{aligned}
\text { L.H.S. }= & l_{m}\left(n-1, k_{0}, \ldots, k_{j-1}, 0, k_{j+1}, \ldots, k_{m-2}\right) \\
& +\sum_{i=2, i \neq j+2}^{m} l_{m}\left(n-i, k_{0}, \ldots, k_{i-2}-1, \ldots, k_{m-2}\right) \\
= & \frac{1}{k_{0} ! \cdots k_{j-1} ! k_{j+1} ! \cdots k_{m-2} !} \\
& \times\left[\begin{array}{c}
(n-1)\left(n-k_{0}-2 k_{1}-\cdots-j k_{j-1}-(j+1) k_{j+1}-(m-1) k_{m-2}-2\right) \\
\left(n-k_{0}-2 k_{1}-\cdots-j k_{j-1}-(j+2) k_{j+1}-\cdots-(m-1) k_{m-2}-3\right) \\
\cdots
\end{array}\right) \\
& \left.+\sum_{i=2, i \neq j+2}^{\left.m-2 k_{0}-3 k_{1}-\cdots-(j+1) k_{j-1}-(j+3) k_{j+1}-\cdots-m k_{m-2}\right)}\right]
\end{aligned}
$$




$$
\begin{aligned}
& \times\left[\begin{array}{c}
(n-i)\left(n-k_{0}-2 k_{1}-\cdots-(i-1) k_{i-2}-\cdots-(m-1) k_{m-2}-2\right) \\
\left(n-k_{0}-2 k_{1}-\cdots-(i-1) k_{i-2}-\cdots-(m-1) k_{m-2}-3\right) \\
\cdots \\
\left(n-2 k_{0}-3 k_{1}-\cdots-(j+1) k_{j-1}-(j+3) k_{j+1}-\cdots-m k_{m-2}+1\right)
\end{array}\right] \\
& =\frac{1}{\left(k_{0}-1\right) ! \cdots\left(k_{j-1}-1\right) !\left(k_{j+1}-1\right) ! \cdots\left(k_{m-2}-1\right) !} \\
& \times\left[\begin{array}{c}
\left(n-k_{0}-2 k_{1}-\cdots j k_{j-1}-(j+2) k_{j+1}-\cdots-(m-1) k_{m-2}-2\right) \\
\left(n-k_{0}-2 k_{1}-\cdots-j k_{j-1}-(j+2) k_{j+1}-\cdots-(m-1) k_{m-2}-3\right) \\
\cdots \\
\left(n-2 k_{0}-3 k_{1}-\cdots-(j+1) k_{j-1}-(j+3) k_{j+1}-\cdots-m k_{m-2}+1\right)
\end{array}\right] \\
& \times\left[\begin{array}{c}
\frac{(n-1)\left(n-2 k_{0}-3 k_{1}-\cdots-(j+1) k_{j-1}-(j+3) k_{j+1} \cdots-m k_{m-2}\right)}{k_{0} k_{1} \cdots k_{j-1} k_{j+1} \cdots k_{m-2}} \\
+\frac{n-2}{k_{1} k_{2} \cdots k_{j-1} k_{j+1} \cdots k_{m-2}}+\frac{n-3}{k_{0} k_{2} \cdots k_{j-1} k_{j+1} \cdots k_{m-2}}+\cdots \\
+\frac{n-m}{k_{0} k_{1} \cdots k_{j-1} k_{j+1} \cdots k_{m-3}}
\end{array}\right] \\
& =\frac{1}{k_{0} ! k_{1} ! \cdots k_{j-1} ! k_{j+1} ! \cdots k_{m-2} !} \\
& \times\left[\begin{array}{c}
n\left(n-k_{0}-2 k_{1}-\cdots-j k_{j-1}-(j+2) k_{j+1}-\cdots-(m-1) k_{m-2}-1\right) \\
\left(n-k_{0}-2 k_{1}-\cdots-j k_{j-1}-(j+2) k_{j+1}-\cdots-(m-1) k_{m-2}-2\right) \\
\cdots \\
\left(n-2 k_{0}-3 k_{1}-\cdots-(j+1) k_{j-1}-(j+3) k_{j+1}-\cdots-m k_{m-2}+1\right)
\end{array}\right] \\
& =l_{m}\left(n, k_{0}, k_{1}, \cdots, k_{j-1}, j k_{j+1}, \cdots, k_{m-2}\right) \\
& =\text { R.H.S. }
\end{aligned}
$$

For other possibilities of more than one $k_{i}=0$, the proof is analogous to the previous case or follows from the induction hypothesis. The following takes care of the case when each $k_{i} \geq 1$ :

$$
\begin{aligned}
& \frac{(n-1)\left(n-2 k_{0}-3 k_{1}-4 k_{2}-\cdots-m k_{m-2}\right)}{k_{0} k_{1} k_{2} \cdots k_{m-2}}+\frac{n-2}{k_{1} k_{2} \cdots k_{m-2}} \\
& +\frac{n-3}{k_{0} k_{2} k_{3} \cdots k_{m-2}}+\cdots+\frac{n-m}{k_{0} k_{1} k_{2} \cdots k_{m-3}} \\
& =\frac{1}{k_{0} k_{1} k_{2} \cdots k_{m-2}}\left[\begin{array}{c}
\left(n^{2}-2 n k_{0}-3 n k_{1}-4 n k_{2}-\cdots-m n k_{m-2}\right) \\
+\left(-n+2 k_{0}+3 k_{1}+4 k_{2}+\cdots+m k_{m-2}\right) \\
n k_{0}-2 k_{0}+n k_{1}-3 k_{1}+\cdots+n k_{m-2}-m k_{m-2}
\end{array}\right] \\
& =\frac{n\left(n-k_{0}-2 k_{1}-3 k_{2}-\cdots-(m-1) k_{m-2}-1\right)}{k_{0} k_{1} k_{2} \cdots k_{m-2}}
\end{aligned}
$$

Proof of the Theorem 2.1. Let $\lambda_{1}, \lambda_{2}, \cdots, \lambda_{m}$ be the eigenvalues of $A$. We prove theorem by mathematical induction on the power of the matrix, that is, $n$. For $n=1$, it is trivial and for $n=2$,

$$
\begin{aligned}
\operatorname{Tr}\left(A^{2}\right) & =\lambda_{1}^{2}+\lambda_{2}^{2}+\cdots+\lambda_{m}^{2} \\
& =\left(\lambda_{1}+\lambda_{2}+\cdots+\lambda_{m}\right)^{2}-2 \sum_{i \neq j} \lambda_{i} \lambda_{j} \\
& =S_{1}^{2}-2 S_{2} .
\end{aligned}
$$

In the similar way, the direct computation using the manipulation of eigenvalues yields the proof of the identity (2.4) for $3 \leq n \leq m-1$. Henceforth we assume that (2.4) holds 
for any positive integer less than $n$, where $n \geq m$. The characteristic equation of $A$ is

$$
\lambda^{m}-S_{1} \lambda^{m-1}+S_{2} \lambda^{m-2}-S_{3} \lambda^{m-3}+\cdots+(-1)^{m} S_{m}=0 .
$$

This, in turn, by the Cayley-Hamilton theorem implies the following:

$$
A^{m}-S_{1} A^{m-1}+S_{2} A^{m-2}-S_{3} A^{m-3}+\cdots+(-1)^{m} S_{m} I=0 .
$$

The trace being a linear operator, gives, a recursive relation,

$$
\begin{aligned}
& \operatorname{Tr}\left(A^{n}\right)=S_{1} \operatorname{Tr}\left(A^{n-1}\right)-S_{2} \operatorname{Tr}\left(A^{n-2}\right)+S_{3} \operatorname{Tr}\left(A^{n-3}\right)-\cdots-(-1)^{m} S_{m} \operatorname{Tr}\left(A^{n-m}\right) \\
& =\sum_{k_{j} \geq 0} \sum_{k_{0}=0}^{\left\lfloor\frac{n-1-3 k_{1}-4 k_{2}-\cdots-m k_{m-2}}{2}\right\rfloor}\left[\begin{array}{c}
(-1)^{k_{0}+k_{2}+k_{4}+\cdots+k}\left\lfloor\frac{m-2}{2}\right\rfloor \\
l_{m}\left(n-1, k_{0}, \cdots, k_{m-2}\right) \\
S_{1}^{n-2 k_{0}-3 k_{1}-\cdots-m k_{m-2}} S_{2}^{k_{0}} \cdots S_{m}^{k_{m-2}}
\end{array}\right] \\
& +\sum_{k_{j} \geq 0} \sum_{k_{0}=1}^{\left\lfloor\frac{n-2-3 k_{1}-4 k_{2}-\cdots-m k_{m-2}}{2}+1\right\rfloor}\left[\begin{array}{c}
(-1)^{k_{0}+k_{2}+k_{4}+\cdots+k}\left\lfloor\frac{m-2}{2}\right\rfloor \\
l_{m}\left(n-2, k_{0}-1, k_{1} \cdots, k_{m-2}\right) \\
S_{1}^{n-2 k_{0}-3 k_{1}-\cdots-m k_{m-2}} S_{2}^{k_{0}} \cdots S_{m}^{k_{m-2}}
\end{array}\right] \\
& +\sum_{\substack{k_{j} \geq 0 \\
k_{1} \geq 1}}^{\left\lfloor\frac{n-3 k_{1}-4 k_{2}-\cdots-m k_{m-2}}{2}\right\rfloor}\left[\begin{array}{c}
(-1)^{k_{0}+k_{2}+k_{4}+\cdots+k}\left\lfloor\frac{m-2}{2}\right\rfloor \\
k_{0}=0 \\
l_{m}\left(n-3, k_{0}, k_{1}-1, k_{2}, \cdots, k_{m-2}\right) \\
S_{1}^{n-2 k_{0}-3 k_{1}-\cdots-m k_{m-2}} S_{2}^{k_{0}} \cdots S_{m}^{k_{m-2}}
\end{array}\right] \\
& +\cdots \\
& +\sum_{\substack{k_{j} \geq 0 \\
k_{m-2} \geq 1}}^{\left\lfloor\frac{n-3 k_{1}-4 k_{2}-\cdots-m k_{m-2}}{2}\right\rfloor} \sum_{k_{0}=0}\left[\begin{array}{c}
(-1)^{k_{0}+k_{2}+k_{4}+\cdots+k}\left\lfloor\frac{m-2}{2}\right\rfloor \\
l_{m}\left(n-m, k_{0}, \cdots, k_{m-3}, k_{m-2}-1\right) \\
S_{1}^{n-2 k_{0}-3 k_{1}-\cdots-m k_{m-2}} S_{2}^{k_{0}} \cdots S_{m}^{k_{m-2}}
\end{array}\right] .
\end{aligned}
$$

Taking certain terms out of the summations and using Lemma 3.3 the theorem follows.

\section{Particular cases}

As the particular cases, we put on record some interesting observations in this section.

Corollary 4.1. For a $2 \times 2$ matrix $A=\left[a_{i j}\right]$,

$$
\operatorname{Tr}\left(A^{n}\right)=\sum_{k_{0}=0}^{\left\lfloor\frac{n}{2}\right\rfloor}(-1)^{k_{0}} l_{2}\left(n, k_{0}\right)[\operatorname{Tr}(A)]^{n-2 k_{0}}[\operatorname{det}(A)]^{k_{0}} .
$$

The following is an interesting fact stating that power and trace commute in case of a singular matrix.

Corollary 4.2. If $A$ is a singular matrix, then $\operatorname{Tr}\left(A^{n}\right)=[\operatorname{Tr}(A)]^{n}$.

Corollary 4.3. If $\operatorname{Tr}(A)=0$, then

$$
\operatorname{Tr}\left(A^{n}\right)= \begin{cases}2(-1)^{\frac{n}{2}}[\operatorname{det}(A)]^{\frac{n}{2}}, & \text { if } n \text { is even; } \\ 0, & \text { if } n \text { is odd. }\end{cases}
$$

Corollary 4.4. If $\operatorname{Tr}(A)=0=\operatorname{det}(A)$, then $\operatorname{Tr}\left(A^{n}\right)=0$.

Now, we apply our scheme of computation to a block matrix. It's noteworthy that in statistics block matrices play a crucial role. 
Corollary 4.5. For a block matrix $A$ of order $2 m$ of the type

$$
\begin{aligned}
& A=\left[\begin{array}{cccc}
A_{1} & & \cdots & 0 \\
& A_{2} & & \vdots \\
\vdots & & \ddots & \\
0 & \cdots & & A_{m}
\end{array}\right] \\
& \operatorname{Tr}\left(A^{n}\right)=\sum_{r=1}^{m} \sum_{k_{0}=0}^{\left\lfloor\frac{n}{2}\right\rfloor}(-1)^{k_{0}} l_{2}\left(n, k_{0}\right)\left[\operatorname{Tr}\left(A_{r}\right)\right]^{n-2 k_{0}}\left[\operatorname{det}\left(A_{r}\right)\right]^{k_{0}} \text {. } \\
& \text { Proof. Clearly } A^{n}=\left[\begin{array}{cccc}
A_{1}^{n} & & \cdots & 0 \\
& A_{2}^{n} & & \vdots \\
\vdots & & \ddots & \\
0 & \cdots & & A_{m}^{n}
\end{array}\right] \text { for all } n \in \mathbb{N} \text {. Consequently, } \\
& \operatorname{Tr}\left(A^{n}\right)=\sum_{r=1}^{m} \operatorname{Tr}\left(A_{r}^{n}\right) \\
& =\sum_{r=1}^{m} \sum_{k_{0}=0}^{\left\lfloor\frac{n}{2}\right\rfloor}(-1)^{k_{0}} l_{2}\left(n, k_{0}\right)\left[\operatorname{Tr}\left(A_{r}\right)\right]^{n-2 k_{0}}\left[\operatorname{det}\left(A_{r}\right)\right]^{k_{0}} .
\end{aligned}
$$

The following is an analogue of [3, Theorem 2.10].

Proposition 4.6. If $A=\left[\begin{array}{ll}a & b \\ b & c\end{array}\right]$, with $a, b, c \geq 0$, then $2 \operatorname{Tr}\left(A^{3}\right) \geq \operatorname{Tr}(A) \cdot \operatorname{Tr}\left(A^{2}\right)$.

Proof.

$$
\begin{aligned}
2 \operatorname{Tr}\left(A^{3}\right)-\operatorname{Tr}(A) \cdot \operatorname{Tr}\left(A^{2}\right)= & 2[\operatorname{Tr}(A)]^{3}-6 \operatorname{Tr}(A) \operatorname{det}(A) \\
& -[\operatorname{Tr}(A)]^{3}+2 \operatorname{Tr}(A) \operatorname{det}(A) \\
= & \operatorname{Tr}(A)\left[[\operatorname{Tr}(A)]^{2}-4 \operatorname{det}(A)\right] \\
= & \operatorname{Tr}(A)\left[(a+c)^{2}-4\left(a c-b^{2}\right)\right] \\
= & \operatorname{Tr}(A)\left[(a-c)^{2}+4 b^{2}\right] \geq 0 .
\end{aligned}
$$

\section{Trace of a negative power of $A$}

The analogue of the formula (2.4) also holds for the trace of negative powers. We limit ourselves to the matrices of order $2 \times 2$, and hence, $A$ will denote a $2 \times 2$ matrices throughout the rest. The proof is on the same line following Lemma 3.3. The proofs are either direct evidence of the results in the previous sections or an obvious workout. From the characteristic equation and the linearity of the trace, we have

$$
\operatorname{Tr}\left(A^{n}\right)=\frac{1}{\operatorname{det}(A)}\left[\operatorname{Tr}(A) \operatorname{Tr}\left(A^{n+1}\right)-\operatorname{Tr}\left(A^{n+2}\right)\right] .
$$

For different values of $n$ in (5.1), we have the following

$$
\operatorname{Tr}\left(A^{-1}\right)=\frac{\operatorname{Tr}(A)}{\operatorname{det}(A)}
$$




$$
\begin{aligned}
& \operatorname{Tr}\left(A^{-2}\right)=\frac{1}{[\operatorname{det}(A)]^{2}}\left[[\operatorname{Tr}(A)]^{2}-2 \operatorname{det}(A)\right] \\
& \operatorname{Tr}\left(A^{-3}\right)=\frac{1}{[\operatorname{det}(A)]^{3}}\left[[\operatorname{Tr}(A)]^{3}-3 \operatorname{Tr}(A) \operatorname{det}(A)\right] \\
& \operatorname{Tr}\left(A^{-4}\right)=\frac{1}{[\operatorname{det}(A)]^{4}}\left[[\operatorname{Tr}(A)]^{4}-4[\operatorname{Tr}(A)]^{2} \operatorname{det}(A)+2[\operatorname{det}(A)]^{2}\right] .
\end{aligned}
$$

We conclude the following on the basis of the above observations.

Theorem 5.1. If $A$ is nonsingular, then

$$
\operatorname{Tr}\left(A^{-n}\right)=\frac{1}{[\operatorname{det}(A)]^{n}} \sum_{k_{0}=0}^{\left\lfloor\frac{n}{2}\right\rfloor}(-1)^{k_{0}} l_{2}\left(n, k_{0}\right)[\operatorname{Tr}(A)]^{n-2 k_{0}}[\operatorname{det}(A)]^{k_{0}} .
$$

Proof. Follows from Lemma 3.3.

Corollary 5.2. If $A$ is nonsingular and $\operatorname{Tr}(A)=0$, then

$$
\operatorname{Tr}\left(A^{n}\right)= \begin{cases}\frac{2(-1)^{\frac{n}{2}}}{[\operatorname{det}(A)]^{\frac{n}{2}}}, & \text { if } n \text { is even; } \\ 0, & \text { if } n \text { is odd. }\end{cases}
$$

Now we obtain the inequality which is completely analogous to the Proposition 4.6.

Proposition 5.3. For a nonsingular matrix $A=\left[\begin{array}{ll}a & b \\ b & c\end{array}\right]$ with $a, b, c \geq 0$,

$$
\begin{array}{ll}
2 \operatorname{Tr}\left(A^{-3}\right) \geq \operatorname{Tr}\left(A^{-1}\right) \cdot \operatorname{Tr}\left(A^{-2}\right) ; \quad \text { if } \operatorname{det}(A)>0 . \\
2 \operatorname{Tr}\left(A^{-3}\right) \leq \operatorname{Tr}\left(A^{-1}\right) \cdot \operatorname{Tr}\left(A^{-2}\right) ; \quad \text { if } \operatorname{det}(A)<0 .
\end{array}
$$

Proof. From (5.2), (5.3) and (5.4),

$$
\begin{aligned}
2 \operatorname{Tr}\left(A^{-3}\right)-\operatorname{Tr}\left(A^{-1}\right) \cdot \operatorname{Tr}\left(A^{-2}\right)= & \frac{2}{[\operatorname{det}(A)]^{3}}\left[[\operatorname{Tr}(A)]^{3}-3 \operatorname{Tr}(A) \operatorname{det}(A)\right] \\
& -\frac{\operatorname{Tr}(A)}{[\operatorname{det}(A)]^{3}}\left[[\operatorname{Tr}(A)]^{2}-2 \operatorname{det}(A)\right] \\
= & \frac{\operatorname{Tr}(A)}{[\operatorname{det}(A)]^{3}}\left[[\operatorname{Tr}(A)]^{2}-4 \operatorname{det}(A)\right] \\
= & \frac{\operatorname{Tr}(A)}{[\operatorname{det}(A)]^{3}}\left[(a-c)^{2}+4 b^{2}\right] .
\end{aligned}
$$

Hence, inequalities (5.5) and (5.6) follow.

Remark 5.4. Similar observations could be made for $3 \times 3$ and even higher order matrices. However, we have limited ourselves to just one order in this note.

Acknowledgment. The author is thankful to the referee for a careful reading and constructive suggestions making the paper readable to more people.

\section{References}

[1] Z. Akyuz and S. Halici, On some combinatorial identities involving the terms of generalized Fibonacci and Lucas sequences, Hacet. J. Math. Stat. 42 (4), 431-435, 2013.

[2] H. Avron, Counting triangles in large graphs using randomized matrix trace estimation, Proceedings of Kdd-Ldmta'10, 2010.

[3] D.J. Karia, K.M. Patil and H.P. Singh, On the sum of powers of square matrices, Oper. Matrices 13 (1), 221-229, 2019. 
[4] J.K. Merikoski, On the trace and the sum of elements of a matrix, Linear Algebra Appl. 60, 177-185, 1984.

[5] V.P. Pugačev, Application of the trace of a matrix to the calculation of its eigenvalues, Ž. Vyčisl. Mat. i Mat. Fiz. 5, 114-116, 1965.

[6] A.V. Zarelua, On congruences for the traces of powers of some matrices, Tr. Mat. Inst. Steklova, 263 (Geometriya, Topologiya i Matematicheskaya Fizika. I), 85-105, 2008 . 